\title{
CALCIUM ION REGULATES AERIAL MYCELIUM FORMATION IN ACTINOMYCETES
}

\author{
Masahiro Natsume, Kazuhisa Yasui ${ }^{\dagger}$ and Shingo Marumo* \\ Department of Agricultural Chemistry, Nagoya University, \\ Chikusa 464-01, Japan
}

(Received for publication September 20, 1988)

\begin{abstract}
$\mathrm{Ca}^{2+}$ induced the formation of aerial mycelia in Streptomyces ambofaciens. Its effect was inhibited by ethylene glycol bis( $\beta$-aminoethyl ether) $-N, N, N^{\prime}, N^{\prime}$-tetraacetic acid, a $\mathrm{Ca}^{2+} \mathrm{specific}^{2}$ chelating agent. A survey of 36 strains of actinomycetes showed that $\mathrm{Ca}^{2+}$ regulated aerial mycelium formation in $21(58 \%)$ of them. The $\mathrm{Ca}^{2+}$ concentration required for aerial mycelium formation in the culture medium ranged from 0.1 to $1.5 \mathrm{~mm}$.
\end{abstract}

Aerial mycelium formation and sporulation are two dramatic morphological events in the life cycle of actinomycetes. These cytodifferentiation processes have been found to be closely related to the production of secondary metabolites such as antibiotics. ${ }^{1,2}$ ) We surveyed actinomycetes in which the formation of aerial mycelium was regulated by endogenous regulating substances and found several those produced aerial mycelium-inducing substances in the culture media. Of these, strains of Streptomyces alboniger and Streptomyces ambofaciens showed activity in a bioassay where their cultured agar discs were separated by a dialysis membrane from the test organisms. Thus the active substances were deduced to be of low molecular weight.

An aerial mycelium-inducing substance (pamamycin-607) was isolated from $S$. alboniger IFO 12738 by a bioassay that used an aerial mycelium-negative mutant of the same strain. ${ }^{3)}$ Pamamycin607 is a novel, sixteen-membered macrodiolide with a dimethylamino group-bearing side chain.4) At $0.1 \mu \mathrm{g} /$ disc, pamamycin-607 induced aerial mycelia in the aerial mycelium-negative mutant.

S. ambofaciens KCC S-0204 formed abundant aerial mycelia on inorganic salts - starch (ISS) agar; but, when grown on yeast extract - malt extract agar (YMA), it produced only substrate mycelia; no aerial mycelium formed. Adding the broth from $S$. ambofaciens KCC S-0204 cultures grown in the ISS medium to substrate mycelia grown on YMA medium induced aerial mycelia. The active substance was purified by ion exchange and cellulose column chromatography, and identified as $\mathrm{Ca}(\mathrm{OAc})_{2}$. It was assumed that the $\mathrm{Ca}^{2+}$ was derived from the $\mathrm{CaCO}_{3}$ which was a component of ISS medium and had been solubilized as $S$. ambofaciens grew and acidified the culture medium.

Recently $\mathrm{Ca}^{2+}$ has been shown to have an important function as a second messenger in animals and plants. ${ }^{52}$ We have investigated the aerial mycelium-inducing activity of $\mathrm{Ca}^{2+}$ in $S$. ambofaciens and we describe here its regulatory function in aerial mycelium formation by this and other actinomycetes.

\section{Materials and Methods}

Microorganisms and Chemicals

The 36 actinomycete species given in Table 2 were obtained from the Institute for Fermentation,

$\dagger$ Present address: Research \& Development Laboratories, Sapporo Breweries Ltd., 10 Okatohme, Yaizu, Shizuoka 425, Japan. 
Osaka and from Kaken Pharmaceutical Co., Ltd.

The $\mathrm{Ca}(\mathrm{OAc})_{2}, \mathrm{CaCl}_{2}, \mathrm{CaCO}_{3}$ and $\mathrm{CoCl}_{2}$ used were of special grade from Wako Pure Chemical Industries, Ltd. Ethylene glycol bis( $\beta$-aminoethyl ether)- $N, N, N^{\prime}, N^{\prime}$-tetraacetic acid (EGTA) was purchased from Dojindo Laboratories.

Yeast extract, malt extract and agar (1st grade) used for YMA medium were purchased from Oriental Yeast Co., Ltd., Difco Laboratories and Katayama Chemical Industries Co., Ltd., respectively.

\section{Culture Media and Conditions}

YMA medium ${ }^{8)}$ was used as the basal medium; its $\mathrm{pH}$ was adjusted to 7.3 before sterilization. A Petri dish (6-cm diameter) containing $7 \mathrm{ml}$ of YMA medium was inoculated with actinomycete species and incubated at $28^{\circ} \mathrm{C}$ for 5 to 7 days.

Calcium salts $\left(\mathrm{Ca}(\mathrm{OAc})_{2}, \mathrm{CaCl}_{2}\right.$ or $\left.\mathrm{CaCO}_{3}\right)$ or EGTA was applied in two ways: 1) A paper disc was impregnated with a sterilized aqueous solution ( $\mathrm{pH}$ of EGTA solution was adjusted to 7.3) and the paper disc was placed on the inoculated agar plate; 2) $\mathrm{Ca}^{2+}$ and/or EGTA was dissolved in the culture medium before $\mathrm{pH}$ adjustment. $\mathrm{CoCl}_{2}$ was applied in the former way.

\section{Quantification of $\mathrm{Ca}^{2+}$ in YMA Medium}

$\mathrm{The} \mathrm{Ca}^{2+}$ concentration of the YMA medium was measured by atomic absorption spectrometry. YMA medium, which was prepared with $1 \mathrm{~N} \mathrm{HCl}$ instead of distilled water, was filtered through Whatman No. 6 filter paper. To the filtrate, strontium chloride $(1 \% \mathrm{w} / \mathrm{v})$ was added to prevent interference by phosphate. ${ }^{3)}$ The $\mathrm{Ca}^{2+}$ concentration of the filtrate was measured with a Hitachi 170$50 \mathrm{~A}$ atomic absorption spectrophotometer at $422.8 \mathrm{~nm}$ with an air-acetylene flame.

\section{Results}

\section{Aerial Mycelium-inducing Activity of $\mathrm{Ca}^{2+}$ in Streptomyces ambofaciens}

The aerial mycelium-inducing activity of $\mathrm{Ca}(\mathrm{OAc})_{2}$ is shown in Fig. 1. At a dose of $2 \mu \mathrm{mol} / \mathrm{disc}$, $\mathrm{Ca}(\mathrm{OAc})_{2}$ produced a grayish zone of aerial mycelia, the diameter of which increased as the dose of $\mathrm{Ca}(\mathrm{OAc})_{2}$ increased. We compared the activities of three $\mathrm{Ca}^{2+}$ salts (Table 1). The two water-

Fig. 1. Aerial mycelium-inducing activity of $\mathrm{Ca}^{2+}$ in Streptomyces ambofaciens KCC S-0204.

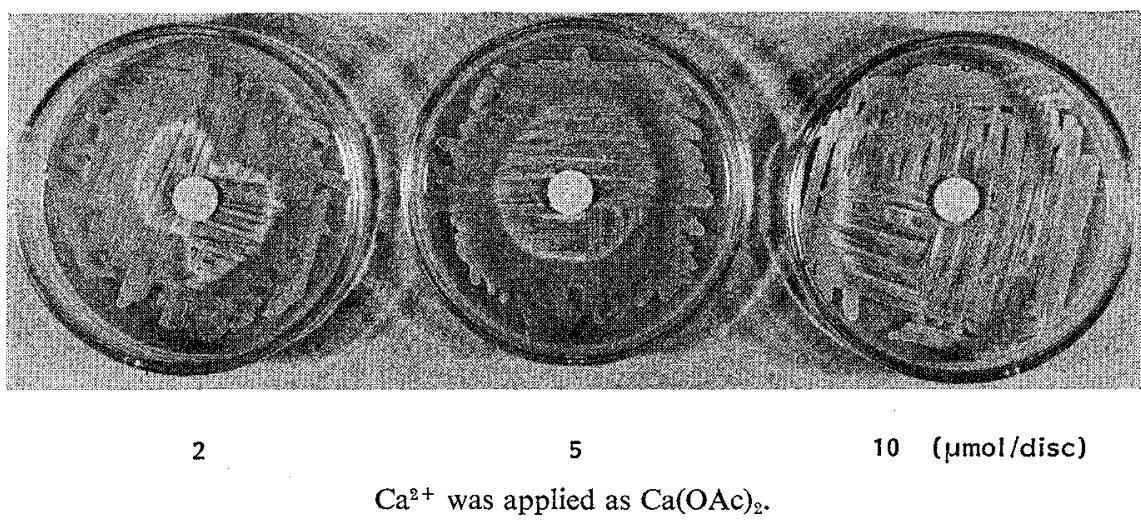

Table 1. Aerial mycelium-inducing activity of calcium salts in Streptomyces ambofaciens.

\begin{tabular}{lcccc}
\hline & $\begin{array}{c}1 \\
(\mu \mathrm{mol} / \mathrm{disc})\end{array}$ & $\begin{array}{c}2 \\
(\mu \mathrm{mol} / \mathrm{disc})\end{array}$ & $\begin{array}{c}5 \\
(\mu \mathrm{mol} / \mathrm{disc})\end{array}$ & $\begin{array}{c}10 \\
(\mu \mathrm{mol} / \mathrm{disc})\end{array}$ \\
\hline $\mathrm{Ca}(\mathrm{OAc})_{2}$ & - & + & + & ++ \\
$\mathrm{CaCl}_{2}$ & - & + & + & ++ \\
$\mathrm{CaCO}_{3}$ & - & - & \pm & \pm \\
\hline
\end{tabular}


soluble salts, $\mathrm{Ca}(\mathrm{OAc})_{2}$ and $\mathrm{CaCl}_{2}$, had similar inducing activities, ranging from 2 to $10 \mu \mathrm{mol} / \mathrm{disc}$, whereas, water-insoluble $\mathrm{CaCO}_{3}$ showed no clear activity even at $10 \mu \mathrm{mol} / \mathrm{disc}$. These results indicate that $\mathrm{Ca}^{2+}$ ion has aerial mycelium-inducing activity. Cultures incubated for more than a week with $\mathrm{CaCO}_{3}$ began to show a narrow zone of aerial mycelium around the paper disc. This delayed activity of $\mathrm{CaCO}_{3}$ is ascribed to its gradual solubilization as $S$. ambofaciens grew, presumably by excreting some acidic metabolites into the medium.

EGTA Inhibits the Aerial Mycelium-inducing Activity of $\mathrm{Ca}^{2+}$ in S. ambofaciens

To confirm that $\mathrm{Ca}^{2+}$ has aerial mycelium-inducing activity, we added EGTA, a $\mathrm{Ca}^{2+}$ specific chelating agent, to $\mathrm{Ca}^{2+}$-containing media at various concentration ratios (Fig. 2). With smaller amounts of EGTA than $\mathrm{Ca}^{2+}$, clear aerial mycelium formation was observed. At equimolar concentrations of $\mathrm{Ca}^{2+}$ and EGTA, only sparse aerial mycelia formed. When EGTA exceeded $\mathrm{Ca}^{2+}$, aerial mycelium formation was inhibited but substrate mycelia grew well. We concluded that $\mathrm{Ca}^{2+}$ is necessary for aerial mycelium formation by S. ambofaciens KCC S-0204.

\section{Effects of $\mathrm{Ca}^{2+}$ and EGTA on Various Actinomycete Species}

We assayed the effect of $\mathrm{Ca}^{2+}$ and EGTA on aerial mycelium formation by 35 actinomycete species. $\mathrm{CaCl}_{2}$ (10 mM) was dissolved in YMA medium. EGTA was applied to the paper disc at a dose of $3 \mu \mathrm{mol} /$ disc. Results showed the actinomycete species to be of four types $(A \sim D)$ (Table 2), typical examples of which are shown in Fig. 3.

\section{Type A}

Strains in which aerial mycelium formation was induced by $\mathrm{Ca}^{2+}$.

Fig. 2. EGTA inhibition of the aerial mycelium-inducing activity of $\mathrm{Ca}^{2+}$ in Streptomyces ambofaciens.

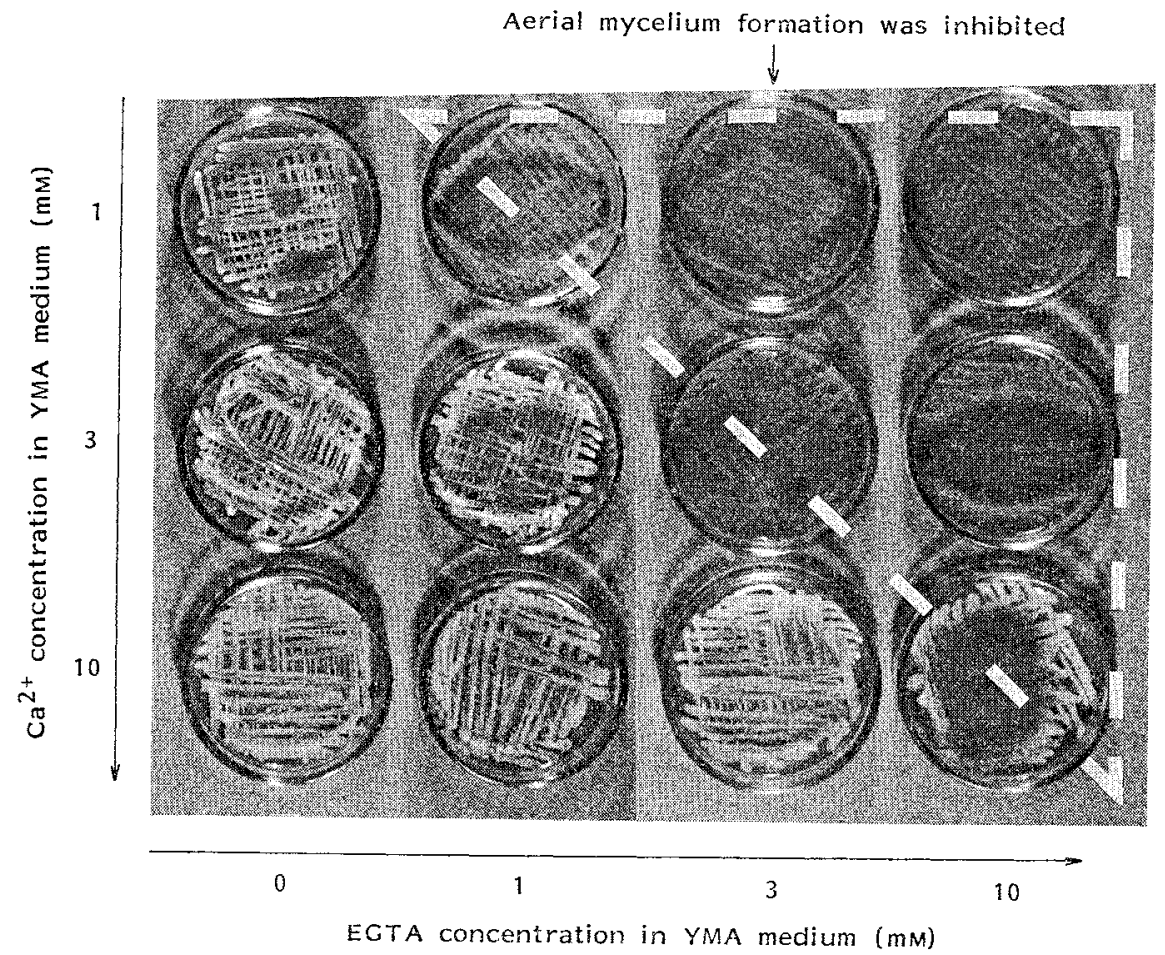


In addition to $S$. ambofaciens KCC S-0204, this group contained $S$. alboniger IFO 12738 . When $\mathrm{Ca}^{2+}$ was added to the medium, the smooth ocherous colonies of $S$. alboniger became powdery white. This $\mathrm{Ca}^{2+}$-induced aerial mycelium formation was inhibited by EGTA as with $S$. ambofaciens.

\section{Type B}

Strains in which aerial mycelium formation was stimulated by $\mathrm{Ca}^{2+}$.

Table 2. Effects of $\mathrm{Ca}^{2+}$ and EGTA on various actinomycete species.

\begin{tabular}{cccc}
\hline & Strain & \multicolumn{2}{c}{ Aerial mycelium formation } \\
\cline { 3 - 4 } Type & YMA & $\begin{array}{c}\text { Induction or } \\
\text { stimulation } \\
\text { by Ca } \mathrm{Ca}^{2+} \\
(10 \mathrm{~mm})\end{array}$ & $\begin{array}{c}\text { Inhibition } \\
\text { by EGTA } \\
(3 \mu \mathrm{mol} / \mathrm{disc})\end{array}$ \\
\hline
\end{tabular}

Type A: Strains in which aerial mycelium formation was induced by $\mathrm{Ca}^{2+}$
Streptomyces alboniger IFO 12738
S. ambofaciens KCC S-0204
$\begin{array}{ll}- & + \\ - & +\end{array}$
Type B: Strains in which aerial mycelium formation was stimulated by $\mathrm{Ca}^{2+}$
Streptomyces griseospiralis KCC S-0869
S. hygroscopicus KCC S-0439
S. kitasatoensis KCC S-1001
S. olivaceus IFO 12805
S. tendae KCC S-0149

$\begin{array}{ll}+ & + \\ + & + \\ + & + \\ + & +\end{array}$

$(+)^{2}$

Type C: Strains in which aerial mycelium formation was inhibited by EGTA

Streptomyces albidoflavus IFO 13010

$S$. argenteolus KCC S-0229

S. fradiae IFO 12773

S. fulvoviridis KCC S- 0374

S. griseus IFO 3102

S. janthinus IFO 12879

S. kanamyceticus IFO 13414

S. kitasatoensis KCC S-1000

S. lavendulae IFO 12789

S. lipmanii IFO 12791

S. rimosus IFO 3228

Saccharopolyspora erythraea IFO 13426

Streptoverticillium albireticuli KCC S-0116

S. griseocarneum IFO 3387

$\begin{array}{ll}+ & - \\ + & - \\ + & - \\ + & - \\ + & - \\ + & - \\ + & - \\ + & - \\ + & - \\ + & - \\ + & - \\ + & -\end{array}$

$+\quad+$

$+\quad+$

$+\quad+$

$+$

$+$

Type D: Strains in which aerial mycelium formation was not affected by addition of $\mathrm{Ca}^{2+}$ or EGTA

Streptomyces humifer IFO 13342

S. glomeroaurantiacus IFO 13380

S. horton IFO 13355

S. lactamdurans IFO 13305

S. luteolutescens IFO 13489

S. olivochromogenes IFO 13067

S. sclerotialus IFO 13356

S. violens IFO 13486

S. aureofaciens IFO 12843

S. cattleya KCC S-0925

S. cirratus KCC S-0738

S. coelicolor IFO 3176

S. eurythermus KCC S-0206

S. flavus KCC S-0036

S. kasugaensis IFO 13851

Number of strains

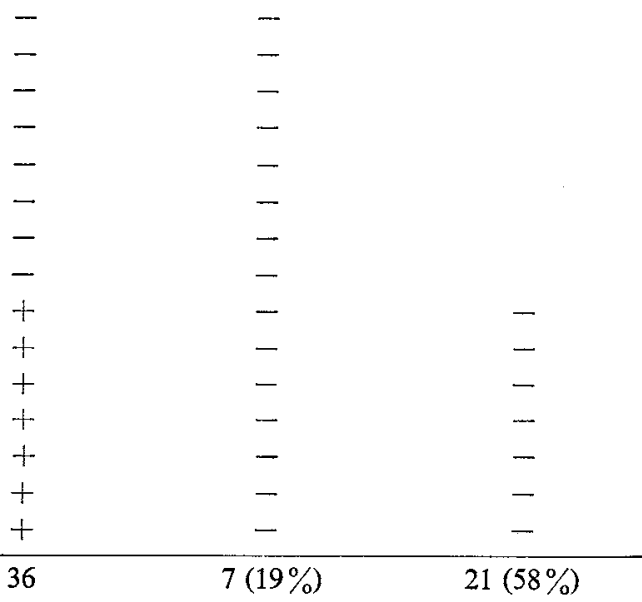

a EGTA inhibited the aerial mycelium formation induced by $\mathrm{Ca}^{2+}$ (see Fig. 2 and text). 
Table 3. Recovery of EGTA inhibition of aerial mycelium formation by excess $\mathrm{Ca}^{2+}$.

\begin{tabular}{|c|c|c|c|}
\hline \multirow[b]{2}{*}{ Strains } & \multicolumn{3}{|c|}{ Aerial mycelium formation } \\
\hline & YMA & $1 \mathrm{mM}$ EGTA & $\begin{array}{c}1 \mathrm{mM} \mathrm{EGTA} \\
5 \mathrm{mM} \mathrm{CaCl}_{2}\end{array}$ \\
\hline Streptomyces albidoflavus IFO 13010 & + & - & + \\
\hline S. argenteolus KCC S-0229 & + & \pm & + \\
\hline S. fradiae IFO 12773 & + & - & + \\
\hline S. fulvoviridis KCC S-0374 & + & - & + \\
\hline S. griseus IFO 3102 & + & \pm & + \\
\hline S. janthinus IFO 12879 & + & \pm & + \\
\hline S. kanamyceticus IFO 13414 & + & - & + \\
\hline S. kitasatoensis KCC S-1000 & + & \pm & + \\
\hline S. lavendulae IFO 12789 & + & - & + \\
\hline S. lipmanii IFO 12791 & + & - & + \\
\hline S. rimosus IFO 3228 & + & - & + \\
\hline Saccharopolyspora erythraea IFO 13426 & + & - & + \\
\hline Streptoverticillium albireticuli KCC S-0116 & + & \pm & + \\
\hline
\end{tabular}

There were 5 strains in this group. Without addition of $\mathrm{Ca}^{2+}$, they produced aerial mycelia sparsely on YMA medium; addition of $\mathrm{Ca}^{2+}$ stimulated aerial mycelium formation. Streptomyces hygroscopicus KCC S-0439 and Streptomyces kitasatoensis KCC S-1001 showed the most marked morphological changes (Fig. 3 ).

As additional evidence that aerial mycelium formation by type $\mathrm{B}$ strains is regulated by $\mathrm{Ca}^{2+}$, EGTA caused inhibition.

\section{Type C}

Strains in which aerial mycelium formation was inhibited by EGTA.

Strains in this group formed abundant aerial mycelia when grown on YMA medium. Addition of $\mathrm{Ca}^{2+}$ had no effect on the appearance of the colonies. However, when EGTA was applied in paper discs, a definite aerial mycelium-inhibitory zone was visible around disc, whereas, substrate mycelia grew well.

Evidence that the inhibition of aerial mycelium formation by EGTA on these type $\mathrm{C}$ strains is the result of $\mathrm{Ca}^{2+}$-starvation was obtained as follows (Table 3): EGTA, added to the medium at $1 \mathrm{~mm}$ inhibited or markedly diminished production of aerial mycelia. This inhibition was removed by excess $(5 \mathrm{~mm}) \mathrm{Ca}^{2+}$. We concluded that aerial mycelium formation by type $\mathrm{C}$ strains also is regulated by $\mathrm{Ca}^{2+}$, bringing the total to 21 (types $\mathrm{A}, \mathrm{B}$ and C) $(58 \%$ ) of the 36 strains tested.

Type D

Other strains. The other 15 strains tested were not induced to form aerial mycelium by $\mathrm{Ca}^{2+}$ nor inhibited by EGTA. Of these, 8 strains formed no aerial mycelium on YMA, ISS, BENNETT's or ATCC sporulation agar media. If we regard these 8 strains as essentially lacking the ability to form aerial mycelium, the percentage of the sporulating strains affected by $\mathrm{Ca}^{2+}$ or EGTA increases to $75 \%$.

\section{$\mathrm{Ca}^{2+}$ Concentration Required for Aerial Mycelium Formation}

Type A strains should require much more $\mathrm{Ca}^{2+}$ than the other types, because they needed extra $\mathrm{Ca}^{2+}$ in addition to that contained in YMA medium. S. alboniger IFO 12738 and S. ambofaciens

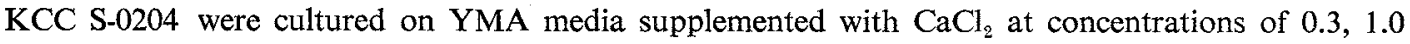


Fig. 3. Effects of $\mathrm{Ca}^{2+}$ and EGTA on various actinomycetes.

Actinomycetes could be classified into four types (the strain type shown in Table 2).

(A) Streptomyces alboniger IFO 12738 (type A)

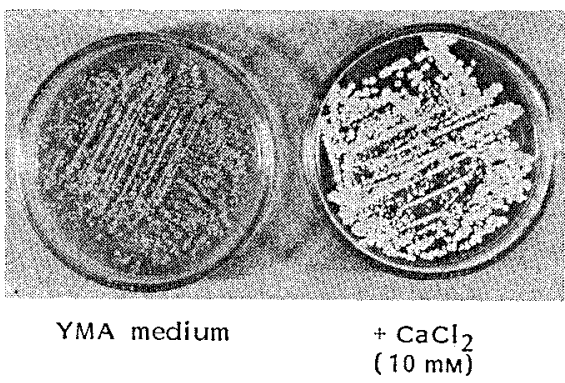

(C) Streptomyces hygroscopicus KCC S-0439 (type B)

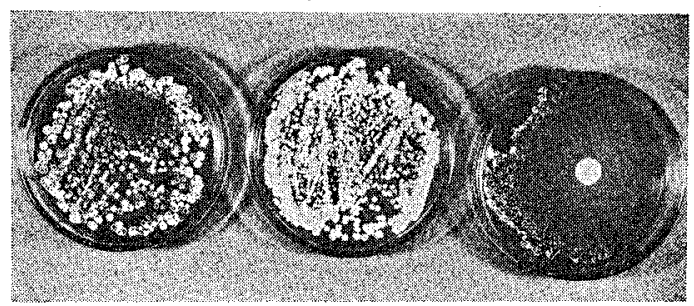

YMA medium

$+\mathrm{CaCl}_{2}$
$(10 \mathrm{mM})$

$(3 \mu \mathrm{mol} / \mathrm{disc})$

(E) Streptomyces lavendulae IFo 12789 (type C)

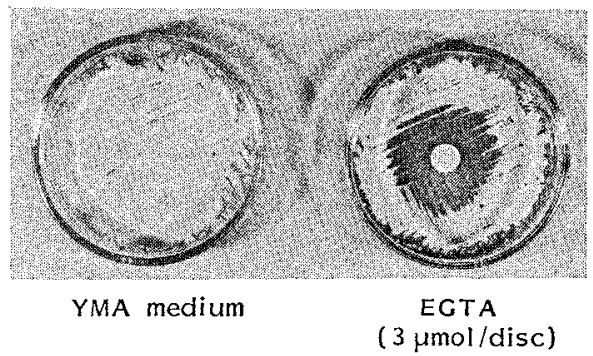

(G) Streptomyces janthinus IFo 12879 (type C)

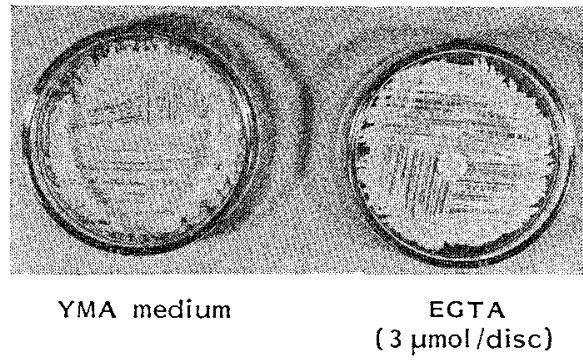

(B) Streptomyces ambofaciens KCC S-0204 (type A)

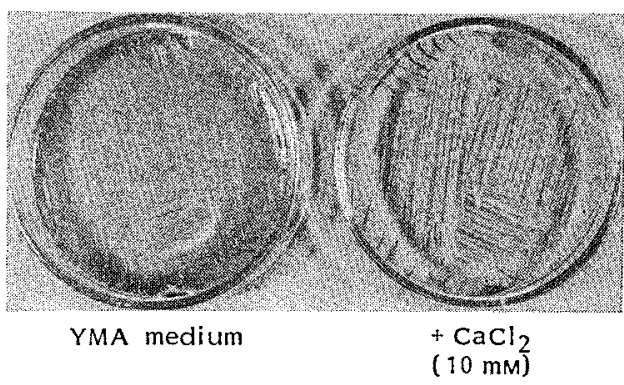

(D) Streptomyces kitasatoensis KCC S-1001 (type B)

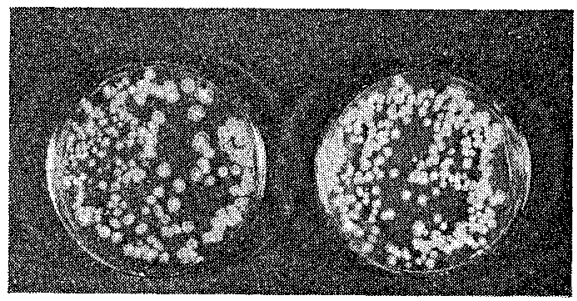

YMA medium

$+\mathrm{CaCl}_{2}$

(10 $\mathrm{mm})$

(F) Streptomyces lipmanii IFO 12791 (type C)

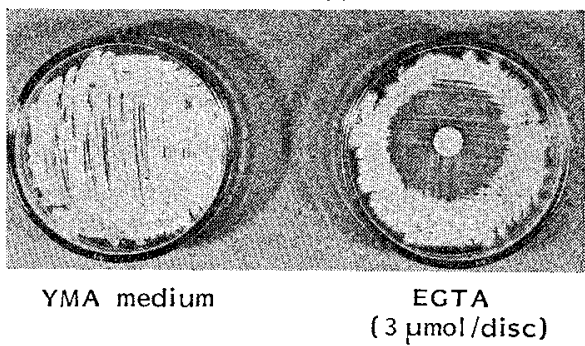

(H) Saccharopolyspora erythraea IFO 13426 (type C)

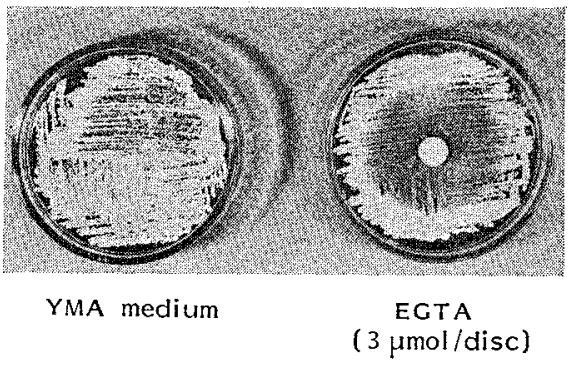

and $3.0 \mathrm{~mm}$. At $1.0 \mathrm{~mm} \mathrm{Ca}{ }^{2+}$, S. alboniger formed aerial mycelia; it required the highest concentration of $\mathrm{Ca}^{2+}$ among the actinomycetes tested (Table 4). The $\mathrm{Ca}^{2+}$ content of YMA medium itself was determined to be $0.5 \mathrm{~mm}$ by atomic absorption analysis. Thus, $1.5 \mathrm{~mm}$ of $\mathrm{Ca}^{2+}$ in the medium 
Table 4. $\mathrm{Ca}^{2+}$ concentrations required for aerial mycelium formation by actinomycetes.

\begin{tabular}{|c|c|c|c|}
\hline \multirow{2}{*}{ Strains } & \multicolumn{3}{|c|}{ Concentration of $\mathrm{CaCl}_{2}(\mathrm{mM})$} \\
\hline & 0.3 & 1.0 & 3.0 \\
\hline \multicolumn{4}{|l|}{ Type A: Induction by $\mathrm{Ca}^{2+}$} \\
\hline Streptomyces alboniger IFO 12738 & - & + & ++ \\
\hline \multirow[t]{3}{*}{ S. ambofaciens KCC S-0204 } & \pm & + & ++ \\
\hline & \multicolumn{3}{|c|}{ Concentration of EGTA (mM) } \\
\hline & 0.2 & 0.4 & 0.8 \\
\hline \multicolumn{4}{|l|}{ Type C: Inhibition by EGTA } \\
\hline Streptomyces fradiae IFO 12773 & ++ & + & - \\
\hline S. fulvoviridis KCC S-0374 & ++ & + & - \\
\hline S. lavendulae IFO 12789 & ++ & ++ & - \\
\hline S. lipmanii IFO 12791 & ++ & $t+$ & - \\
\hline Saccharopolyspora erythraea IFO 13426 & \pm & - & - \\
\hline
\end{tabular}

$\mathrm{Ca}^{2+}$ concentration in YMA medium was determined to be $0.5 \mathrm{mM}$ by atomic absorption analysis.

was required for aerial mycelium formation by S. alboniger IFO 12738.

Since strains of type $\mathrm{C}$ formed abundant aerial mycelia on YMA medium, the $\mathrm{Ca}^{2+}$ present must be sufficient for them. We attempted to immobilize this $\mathrm{Ca}^{2+}$ by adding EGTA at concentrations of $0.2,0.4$ and $0.8 \mathrm{mM}$. Even in the presence of $0.4 \mathrm{mM}$ EGTA, aerial mycelium formation by Streptomyces lavendulae IFO 12789 and Streptomyces lipmanii IFO 12791 was not inhibited. This indicates that $0.1 \mathrm{~mm}$ of $\mathrm{Ca}^{2+}$ is sufficient for aerial mycelium formation by these strains.

Thus, the concentration of $\mathrm{Ca}^{2+}$ in the medium required for aerial mycelium formation ranged from 0.1 to $1.5 \mathrm{mM}$.

\section{Effect of $\mathrm{Co}^{2+}$ on Actinomycete Species}

We also examined the effect of $\mathrm{Co}^{2+}$ on aerial mycelium formation by the paper disc method. At 1 and $5 \mu \mathrm{mol} / \mathrm{disc}, \mathrm{Co}^{2+}$ induced aerial mycelium formation by $S$. ambofaciens $\mathrm{KCC}$ S-0204 and stimulated that by $S$. kitasatoensis KCC S-1001, and it also restored aerial mycelium formation after EGTA-inhibition $(1 \mathrm{mM})$ in 5 of the type $\mathrm{C}$ strains. The definite difference between $\mathrm{Co}^{2+}$ and $\mathrm{Ca}^{2+}$ was that $\mathrm{Co}^{2+}$ caused growth inhibition around the paper disc at a dose required for aerial mycelium formation. At $1 \mu \mathrm{mol} / \mathrm{disc}, S$. ambofaciens formed a growth inhibitory zone (1.6-cm diameter) around the paper disc, the outer area of which was an aerial mycelium-inducing zone $(0.8 \mathrm{~cm}$ in width). At $5 \mu \mathrm{mol} / \mathrm{disc}, \mathrm{Co}^{2+}$ caused growth inhibition around the disc in all the strains tested.

\section{Discussion}

Cytodifferentiation of actinomycetes is regulated by endogenous substances such as A-factor ${ }^{83}$ and factor- $\mathrm{C}^{9}$. MCCANN and Pogell showed that $S$. alboniger produced the regulating substance, pamamycin, which they obtained as a mixture of four homologues (MWs 621, 635, 649 and 663). ${ }^{10)}$ We isolated a new pamamycin homologue, pamamycin-607 (MW 607) in a pure form and elucidated its structure. ${ }^{3,4)}$

In the culture media used for growth of actinomycetes, $\mathrm{CaCO}_{3}$ is frequently one of the components. Its role hitherto was considered to be maintaining neutral $\mathrm{pH}$ conditions. Our present research, however, has shown that $\mathrm{CaCO}_{3}$ has another function; induction or stimulation of cytodifferentiation such as aerial mycelium formation.

HICKEY and TRESNER reported that $\mathrm{Co}^{2+}$ improved the rate and extent of sporulation of Streptomyces species. ${ }^{11)}$ We examined the effect of $\mathrm{Co}^{2+}$ on aerial mycelium formation. Aerial mycelium 
formation of 7 strains was effected by $\mathrm{Co}^{2+}$, but growth of all the strains tested was inhibited at a same dose required for aerial mycelium formation. This result may indicate that aerial mycelium formation by $\mathrm{Co}^{2+}$ is an indirect effect of growth inhibition.

Recently the intracellular $\mathrm{Ca}^{2+}$ concentration of Escherichia coli was measured by GANGOLA and RoseN ${ }^{12)}$; when the $\mathrm{Ca}^{2+}$ concentration in the medium was increased from 0.08 to $10 \mathrm{mM}$, the concentration of free $\mathrm{Ca}^{2+}$ in the cells was maintained at $0.1 \mu \mathrm{M}$, although the total amount of $\mathrm{Ca}^{2+}$ increased in parallel with the extracellular $\mathrm{Ca}^{2+}$. With actinomycetes we have now shown that the extracellular $\mathrm{Ca}^{2+}$ concentration required for aerial mycelium formation ranges from 0.1 to $1.5 \mathrm{~mm}$. This is within the range of $\mathrm{Ca}^{2+}$ in the medium used for $E$. coli by GANGOLA and RoSEN. We suggest that the free $\mathrm{Ca}^{2+}$ concentration in actinomycetes cells is probably comparable to that in $E$. coli cells.

From its macrodiolide structure, we expected that pamamycin-607 might be a $\mathrm{Ca}^{2+}$ transporter. However, this could not be demonstrated by in vitro two phase partition experiments. ${ }^{8)}$ Examination of CPK space filling atomic models indicates that the sixteen-membered macrodiolide ring of pamamycin-607 has insufficient space to incorporate $\mathrm{Ca}^{2+}$. However, we found that pamamycin-607 has anion transporting ability; in its presence $\mathrm{MnO}_{4}{ }^{-}$and $\mathrm{Cl}^{-}$transfer from water to the benzene layer. ${ }^{3)}$ CHOw and Pogell reported that one of the primary target of pamamycins in growth inhibition of bacteria was inhibition of phosphate transport. ${ }^{13)}$ As one possible explanation why pamamycin-607 and $\mathrm{Ca}^{2+}$ exhibit similar activity in $S$. alboniger, $\mathrm{Ca}^{2+}$ might lessen the intracellular concentration of phosphate by forming insoluble calcium phosphate; sequestration of phosphate in the cells could be related to aerial mycelium formation.

Since $\mathrm{Ca}^{2+}$ showed activity in a wide range of actinomycete species, the mode of action of $\mathrm{Ca}^{2+}$ in aerial mycelium formation is of great interest.

\section{References}

1) Redshaw, P. A.; P. M. McCann, M. A. Pentella \& B. M. Pogell: Simultaneous loss of multiple differentiation functions in aerial mycelium-negative isolates of streptomycetes. J. Bacteriol. 127: 891 899,1979

2) GinTHER, C. L.: Sporulation and the production of serine protease and cephamycin C by Streptomyces lactamudurans. Antimicrob. Agents Chemother. 15: 522 526, 1979

3) Kondo, S.; K. Yasur, M. Natsume, M. Katayama \& S. Marumo: Isolation, physico-chemical properties and biological activity of pamamycin-607, an aerial mycelium-inducing substance from Streptomyces alboniger. J. Antibiotics 41: 1196 1204, 1988

4) Kondo, S.; K. Yasur, M. Katayama, S. Marumo, T. Kondo \& H. Hattori: Structure of pamamycin607, an aerial mycelium-inducing substance of Streptomyces alboniger. Tetrahedron Lett. 28: 5861 5864,1987

5) Carafoli, E. \& J. T. Penniston: The calcium signal. Sci. Am. 253: 50 89, 1985

6) Pridham, T. G.; P. Anderson, C. Foley, L. A. Lindenfelser, C. W. Hesseltine \& R. G. Benedict: A selection of media for maintenance and taxonomic study of Streptomyces. In Antibiotics Annual 19561957. Eds., H. Welch \& F. Marti-IbañeZ, pp. 947 953, Medical Encyclopedia, Inc., New York, 1957

7) REeD, P. W. \& H. A. LARDY: A23187: A divalent cation ionophore. J. Biol. Chem. 247: 6970 6977, 1972

8) Khokhlov, A. S.; L. N. Anisova, I. I. Tovarova, F. M. Kleiner, I. V. Kovalenko, O. I. Krasilnikova, E. YA. Kornitskaya \& S. A. Pliner: Effect of A-factor on the growth of asporogenous mutants of Streptomyces griseus, not producing this factor. Z. Allg. Mikrobiol. 13: 647 655, 1973

9) SZABO, G.; T. VAlyi-NAGY \& S. Vitalis: An endogenous factor regulating the life cycle of Streptomyces griseus. Acta Biol. Hung. 18: 237 243, 1967

10) McCann, P. A. \& B. M. Pogell: Pamamycin: A new antibiotic and stimulator of aerial mycelia formation. J. Antibiotics 32: 673 678, 1979

11) HICKey, R, J. \& H. D. TRESNER: A cobalt-containing medium for sporulation of Streptomyces species. J. Bacteriol. 64: $891 \sim 892,1952$

12) Gangola, P. \& B. P. Rosen: Maintenance of intracellular calcium in Escherichia coli. J. Biol. Chem. 262: $12570 \sim 12574,1987$

13) Chow, W.-G. \& B. M. Pogels: Mode of action of pamamycin in Staphylococcus aureus. Antimicrob. Agents Chemother. 20: 443 454, 1981 